\title{
WAVE PROPAGATION IN RODS OF VOIGT MATERIAL AND VISCO-ELASTIC MATERIALS WITH THREE-PARAMETER MODELS*
}

\author{
BY \\ J. A. MORRISON \\ Brown University
}

\begin{abstract}
The problem of an impulsively applied and subsequently maintained constant velocity at the end of a semi-infinite rod of Voigt material is considered and integral expressions are obtained for the velocity and stress distributions in the rod. Apart from a constant factor the stress distribution arising from an impulsively applied constant stress at the end of the rod is the same as the velocity solution above. The same problem is considered also for materials with three-parameter models. The stress distributions for both the case of constant applied stress and constant applied velocity are represented graphically for the materials considered, dimensionless co-ordinates being used.
\end{abstract}

1. Fundamental considerations and the Voigt modei. Lee and Kanter ${ }^{1}$ considered the problem of an impulsively applied constant velocity on the end of a rod of Maxwell material and analytically determined the subsequent stress distribution in the rod. Glauz and $\mathrm{Lee}^{2}$ treated the same problem for a four-parameter model visco-elastic material and determined the stress and velocity distributions by the method of characteristics. In order to complete the picture we consider here the other two-parameter model, which corresponds to the Voigt material, and also the three-parameter models.

The Voigt model is shown in Fig. 1 and is composed of a spring and a dashpot in

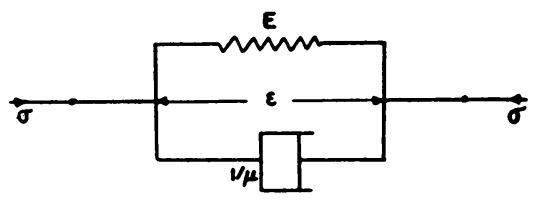

Fig. 1. Model for Voigt material.

parallel and so the Voigt material presents a retarded elastic response to stress. The stress strain relation is

$$
\sigma=E \epsilon+\frac{1}{\mu} \epsilon_{t},
$$

where $\sigma$ is the stress, $\epsilon$ the strain, the suffix denotes differentiation with respect to time and $E$ and $\mu$ are elastic and viscous constants, respectively, for the material.

Let us consider a creep test, together with unloading, in which a constant stress $\sigma_{0}$ is suddenly applied at time $t=0$ and suddenly removed at $t=t_{0}$ so that $\sigma^{\prime}=\sigma_{0}\{H(t)-$ $\left.H\left(t-t_{0}\right)\right\}$ where $H(t)$ is Heavisides' step function,

$$
H(t)=\left\{\begin{array}{l}
1, t>0 \\
0, t<0 .
\end{array}\right.
$$

*Received June 10, 1955. Sponsored under a Department of Defense Contract, NOrd-11496 between the Bureau of Ordnance, Navy Department, and Brown University.

'E. H. Lee and I. Kanter, J. Appl. Phys. 24, 1115-1122 (1953).

${ }^{2}$ R. D. Glauz and E. H. Lee, J. Appl. Phys. 25, 947-953 (1954). 
From Eq. (1) it is found by integration that $\epsilon=\left\{\psi(t) H(t)-\psi\left(t-t_{0}\right) H\left(t-t_{0}\right)\right\}$, where $\psi(t)=\left(\sigma_{0} / E\right)\left(1-e^{-E \mu t}\right)$. For convenience we introduce dimensionless variables $\tau=E \mu t$, $\Sigma^{\prime}=\sigma^{\prime} / \sigma_{0}$ and $\varepsilon=(E \boldsymbol{\epsilon}) / \sigma_{0}$. Then,

where

$$
\begin{aligned}
& \Sigma^{\prime}=\left\{H(\tau)-H\left(\tau-\tau_{0}\right)\right\}, \\
& \mathcal{E}=\left\{\varphi(\tau) H(\tau)-\varphi\left(\tau-\tau_{0}\right) H\left(\tau-\tau_{0}\right)\right\},
\end{aligned}
$$

$$
\varphi(\tau)=\left(1-e^{-\tau}\right) .
$$

The creep test is represented in Fig. 2 where $\tau_{0}=E \mu t_{0}$ has been given the value 2 .

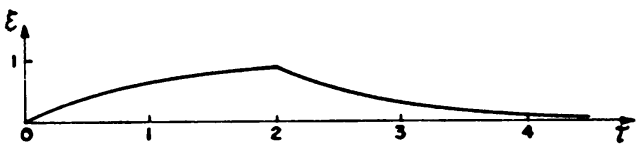

FIG. 2.

We now turn our attention to the problem at hand. We are concerned with the propagation of longitudinal waves in a semi-infinite rod, $x \geq 0$, which is initially unstrained and at rest. Let $u(x, t)$ denote the displacement of the section $x$ of the rod so that its position at time $t$ is given by $(x+u)$. Then, if $\rho$ is the density of the unstrained material,

$$
\begin{aligned}
\rho u_{t} & =-\sigma_{x}, \\
\epsilon & =-u_{x},
\end{aligned}
$$

where suffixes denote partial differentiation with respect to the corresponding variable. Here $\sigma$ and $\epsilon$ are the nominal compressive stress and nominal compressive strain, respectively and it is supposed that the stress strain relation of Eq. (1) applies to these nominal values.

Eliminating $\sigma$ and $\epsilon$ from Eqs. (1), (4) and (5) we obtain

$$
\rho u_{t \imath}=E u_{x x}+\frac{1}{\mu} u_{x x t},
$$

from which it is readily verified that the stress $\sigma$ and the particle velocity $v=u_{t}$ both satisfy the partial differential equation

$$
\rho f_{t t}=E f_{x x}+\frac{1}{\mu} f_{x x t} .
$$

It follows that the stress distribution $\sigma^{\prime}(x, t)$ in the case in which a constant stress $\sigma_{0}$ is suddenly applied at the end of the rod and then maintained is of the same form as the velocity distribution $v(x, t)$ for the case of a suddenly applied and subsequently maintained velocity $v_{0}$ at the end of the rod. In fact,

$$
\frac{v(x, t)}{v_{0}}=\frac{\sigma^{\prime}(x, t)}{\sigma_{0}} \text {. }
$$

If we introduce the dimensionless variables $\xi=(\rho E)^{1 / 2} \mu x, V=v(x, t) / v_{0}$ and, as before, $\tau=E \mu t, \Sigma^{\prime}=\sigma^{\prime}(x, t) / \sigma_{0}$, Eq. (8) becomes

$$
\Sigma^{\prime}(\xi, \tau)=V(\xi, \tau)
$$


We therefore confine our attention to the problem in which the end of the rod (which is initially unstrained and at rest) is suddenly given a velocity $v_{0}$ which is subsequently maintained and determine the stress and velocity solutions, $\sigma(x, t)$ and $v(x, t)$. We introduce the dimensionless stress $\Sigma(\xi, \tau)=\sigma(x, t) /(\rho E)^{1 / 2} v_{0}$. We will then have determined the stress solutions $\Sigma(\xi, \tau)$ and $\Sigma^{\prime}(\xi, \tau)$ both for the case of constant applied velocity and constant applied stress.

The determination of the solutions, for the Voigt material, is carried out in Appendix $A$ by the method of Laplace transforms. $Z_{v e r e v}{ }^{3}$ adopted the same method but carried out the inversion of the Laplace transform in a different manner and obtained an infinite integral expression for the velocity distribution. The present author gave a similar expression for the stress distribution ${ }^{4}$ but the method adopted in this paper is readily extended to the case of the three-parameter model with two viscous elements and in addition the finite integral expressions obtained are readily computed for the moderate values of $\tau$ in which we are interested.

The stress solutions $\Sigma(\xi, \tau)$ and $\Sigma^{\prime}(\xi, \tau)$ as given by Eqs. (A.18) and (A.14), respectively, are depicted graphically in Figs. 3(a) and 3(b). In Fig. 3(a), $\Sigma$ is plotted against

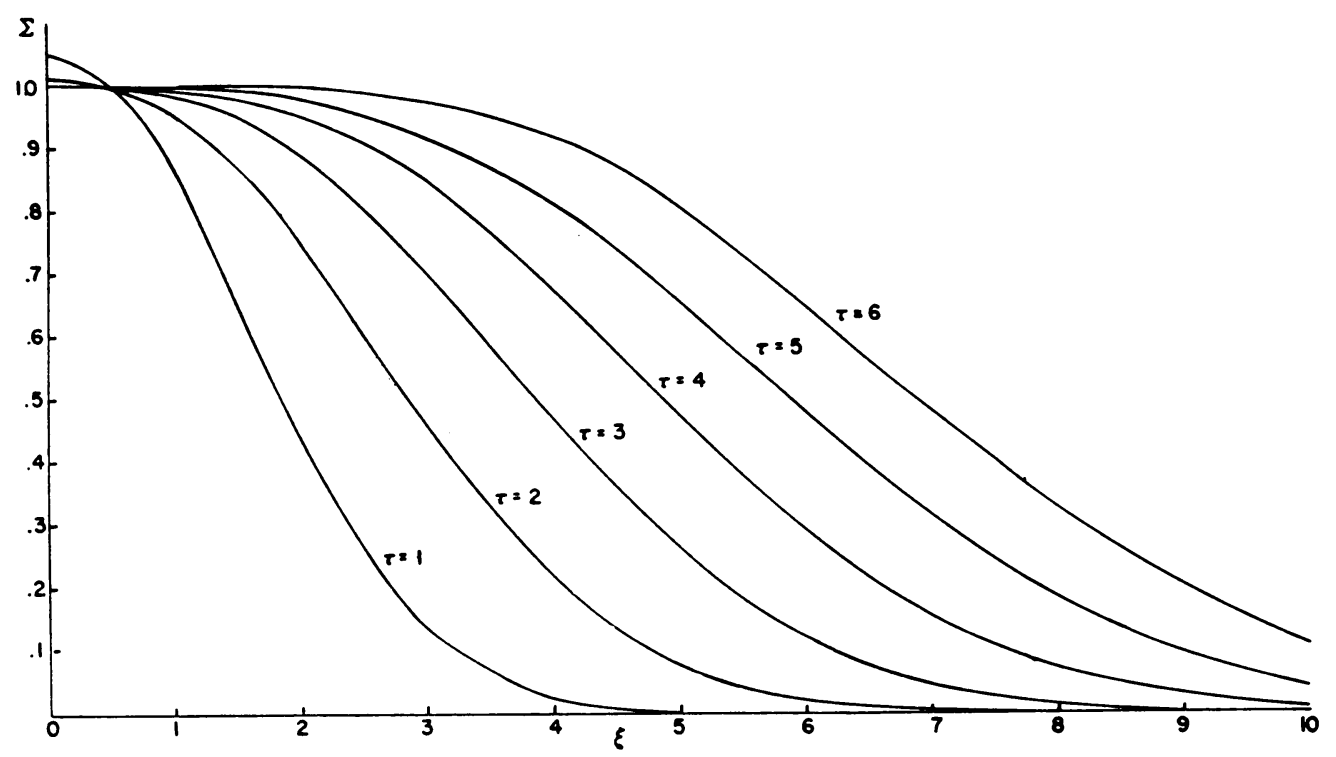

FIG. 3(a). Voigt model.

$\xi$ for different values of $\tau$ so that the distribution of stress in the rod at various times, arising from an impulsively applied and subsequently maintained constant velocity at the end of the rod, is illustrated. From Eq. (A.19) it is seen that the stress at the end of the rod, which is very large immediately after impact, decreases rapidly at first and subsequently continues to decrease, but with diminishing rapidity, ultimately approaching the value unity. The stress at a position of the rod very close to the end rises extremely

'I. N. Zverev, Prik. Mat. Mek. 15, 295-302 (1950), (Russian). (Brown University translation A11-T12/16).

'J. A. Morrison, Wave propagation in a rod of Voigt material, Tech. Rept. PA-5/17, Div. Appl. Math., Brown University, Providence, R. I. (1953). 
rapidly shortly after impact to a large value and then commences to decrease. The occurrence of large stresses near the end of the rod is due to the response of the viscous element to an attempted sudden change in strain. Such a change in strain would correspond to an infinite strain rate and so demand an infinite stress to produce it.

However, as the time increases the viscous behavior tends to die out and the rod tends to behave as in the case of a completely elastic material. At each position in the rod the stress ultimately approaches the value unity. It is noticed that there is a considerable spread in the stress distribution, as opposed to the sharp wave-front occurring in the case of an elastic material, and that the effect of the disturbance is propagated instantaneously (in a decaying manner) to all positions in the rod.

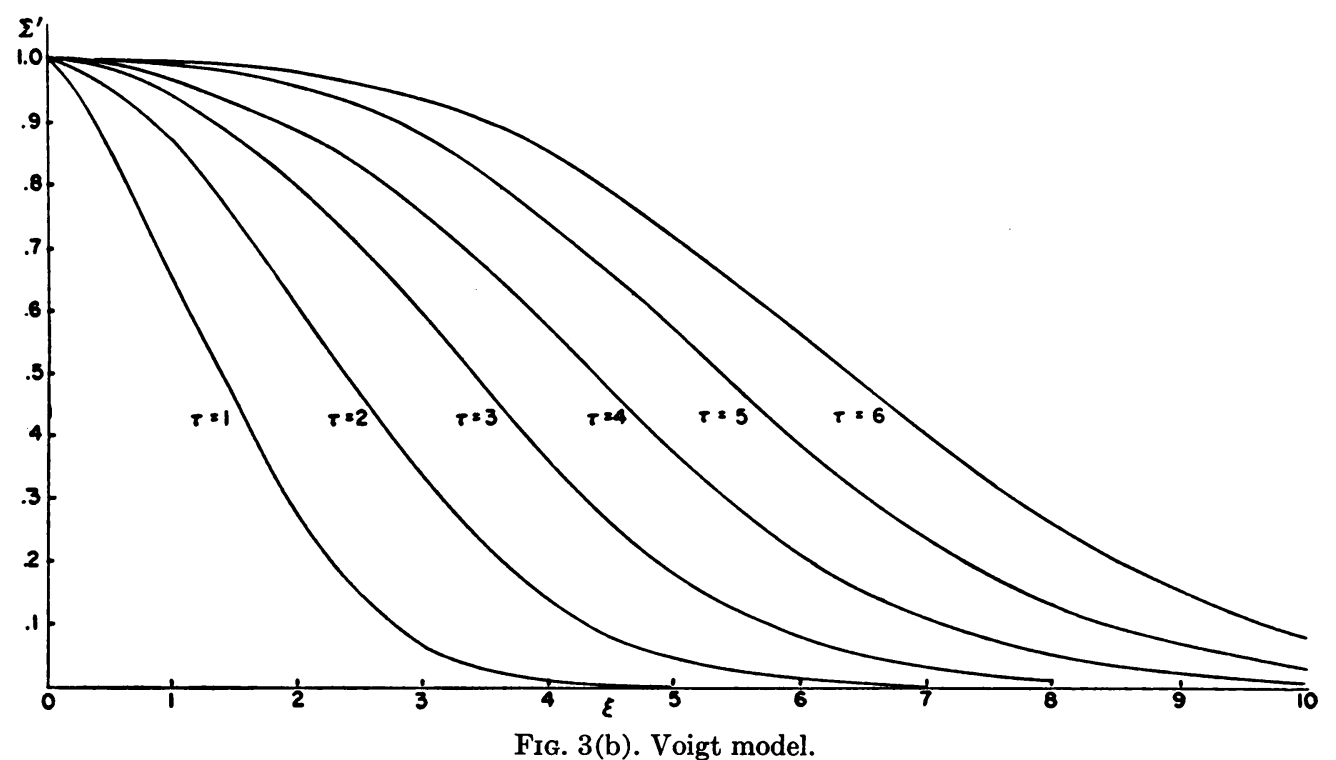

In Fig. 3(b), $\Sigma^{\prime}$ is plotted against $\xi$ for different values of $\tau$ so that the distribution of stress in the rod at various times, arising from an impulsively applied and subsequently maintained constant stress at the end of the rod, is illustrated. At each position in the rod $\Sigma^{\prime}$ ultimately approaches the value unity but it is again interesting to note the considerable spread in the stress distribution.

2. The three-parameter model with one elastic and two viscous elements. There are four true three-parameter models, all the others reducing effectively to models with fewer parameters. We first consider the two models shown in Figs. 4(a) and 4(b) which

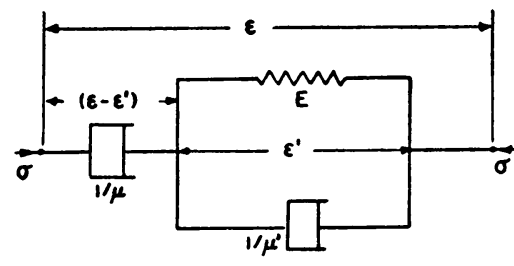

FIG. 4(a).

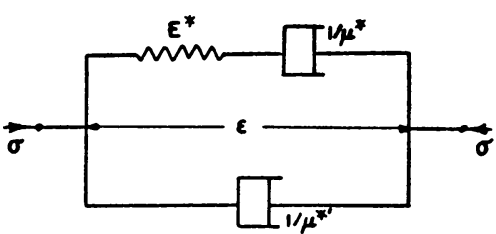

Fig. 4(b). 
have two dashpots and one spring. For the model of Fig. 4(a) we have

$$
E \epsilon^{\prime}+\frac{1}{\mu^{\prime}} \epsilon_{t}^{\prime}=\sigma=\frac{1}{\mu}\left(\epsilon_{t}-\epsilon_{t}^{\prime}\right) \text {. }
$$

Hence, eliminating $\epsilon^{\prime}$,

$$
E \epsilon_{t}+\frac{\epsilon_{t t}}{\mu^{\prime}}=E_{\mu \sigma}+\left(1+\frac{\mu}{\mu^{\prime}}\right) \sigma_{t} .
$$

In a similar manner we obtain the stress strain relation for the model of Fig. 4(b),

$$
\frac{\sigma_{t}}{E^{*}}+\mu^{*} \sigma=\epsilon_{t}\left(1+\frac{\mu^{*}}{\mu^{*^{\prime}}}\right)+\frac{\epsilon_{t t}}{\mu^{* \prime} E^{*}} .
$$

If we set $\left(E / E^{*}\right)^{1 / 2}=\mu^{*} / \mu=\mu^{* \prime} / \mu^{\prime}=\left(1+\mu / \mu^{\prime}\right)$, then Eqs. (11) and (12) become identical. Consequently we confine our attention to the model of Fig. 4(a). If we set $\sigma=\sigma_{0} H(t)$ in Eq. (11) and integrate we find that

$$
\epsilon=\frac{\sigma_{0}}{E}\left[E_{\mu t}+\left(1-e^{-E_{\mu^{\prime}} t}\right)\right] H(t),
$$

so that the creep test with unloading is given by Eq. (2) with

$$
\varphi(\tau)=\left\{\tau+\left(1-e^{-\tau / \lambda}\right)\right\},
$$

where $\lambda=\mu / \mu^{\prime}$. This is depicted graphically in Fig. 5 for the particular case $\lambda=1$.

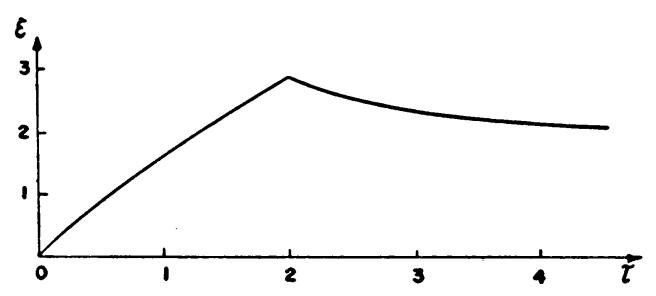

FIG. 5.

It is noted that after the removal of the applied stress the strain approaches an asymptotic value given by $\epsilon=\tau_{0}(=2)$ and this residual strain is due to the viscous flow in the dashpot with viscous constant $\mu$ which is in series with the retarded elastic or Voigt element. The strain of the delayed elastic portion is asymptotically recovered, as is seen from Fig. 2.

Eliminating $\sigma$ and $\epsilon$ from Eqs. (4), (5) and (11) and setting $v=u_{t}$,

$$
\rho E_{\mu v_{t}}+\rho\left(1+\frac{\mu}{\mu^{\prime}}\right) v_{t t}=E v_{x x}+\frac{1}{\mu^{\prime}} v_{x x t},
$$

and it is readily verified that $\sigma$ satisfies the same equation. Hence Eq. (9) again holds and the stress distributions $\Sigma(\xi, \tau)$ and $\Sigma^{\prime}(\xi, \tau)$ arising when the end of a rod, initially unstrained and at rest, of a material corresponding to the three-parameter model of Fig. 4(a) is impulsively subjected to constant applied velocity and constant applied stress, respectively, are determined in Appendix B, Eqs. (B.8) and (B.14). 
$\Sigma$ and $\Sigma^{\prime}$ are plotted in Figs. 6(a) and 6(b), respectively, against $\xi$ for different values of $\tau$ in the particular case $\lambda=1$, so that the two viscous constants in the model are equal. Thus in Fig. 6(a) the stress distribution in the rod at various times, arising from an impulsively applied and subsequently maintained constant velocity at the end of the rod, is illustrated. The stress at the end of the rod is very large immediately after impact due to the combined "rigid" responses of the viscous element and delayed elastic element, in series, which comprise the model of Fig. 4(a). Nevertheless it decreases very rapidly and has a value less than unity at time $\tau=1$. The stress at the end of the rod continues

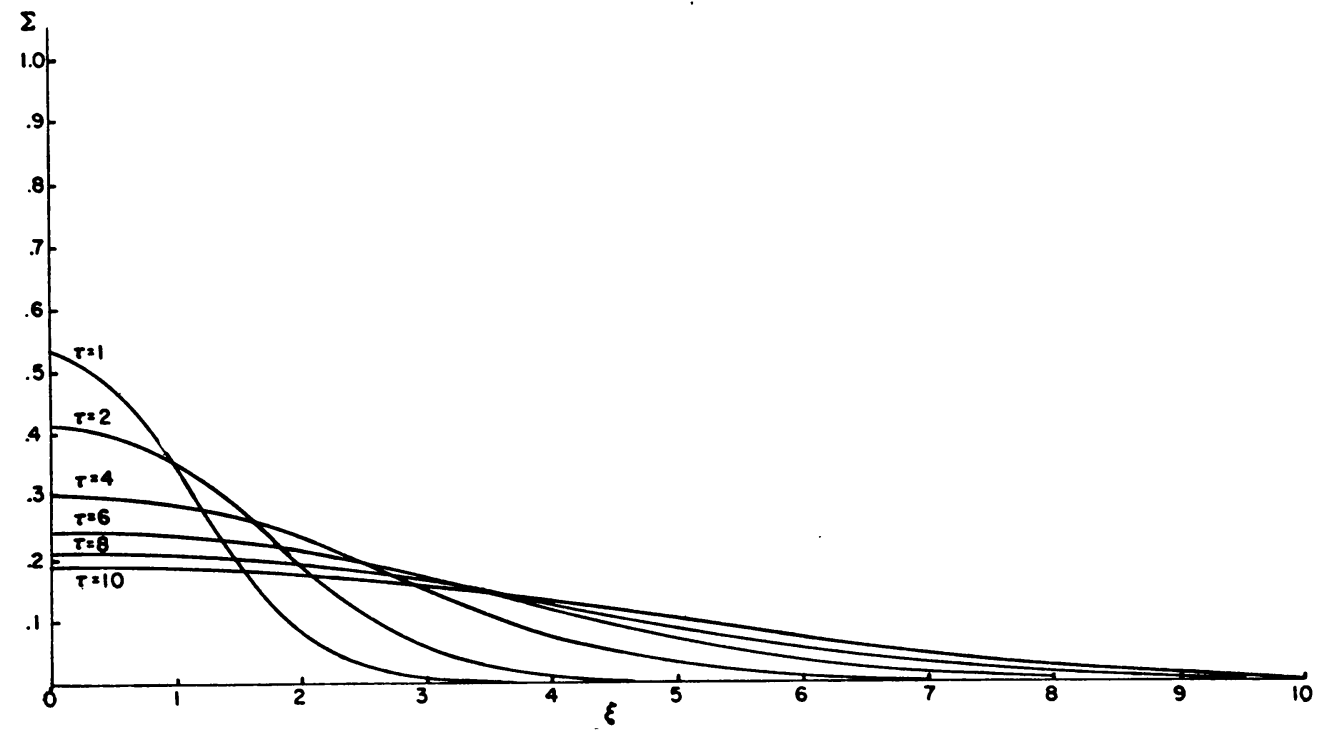

FIG. 6(a). Three parameter (1 elastic, 2 viscous).

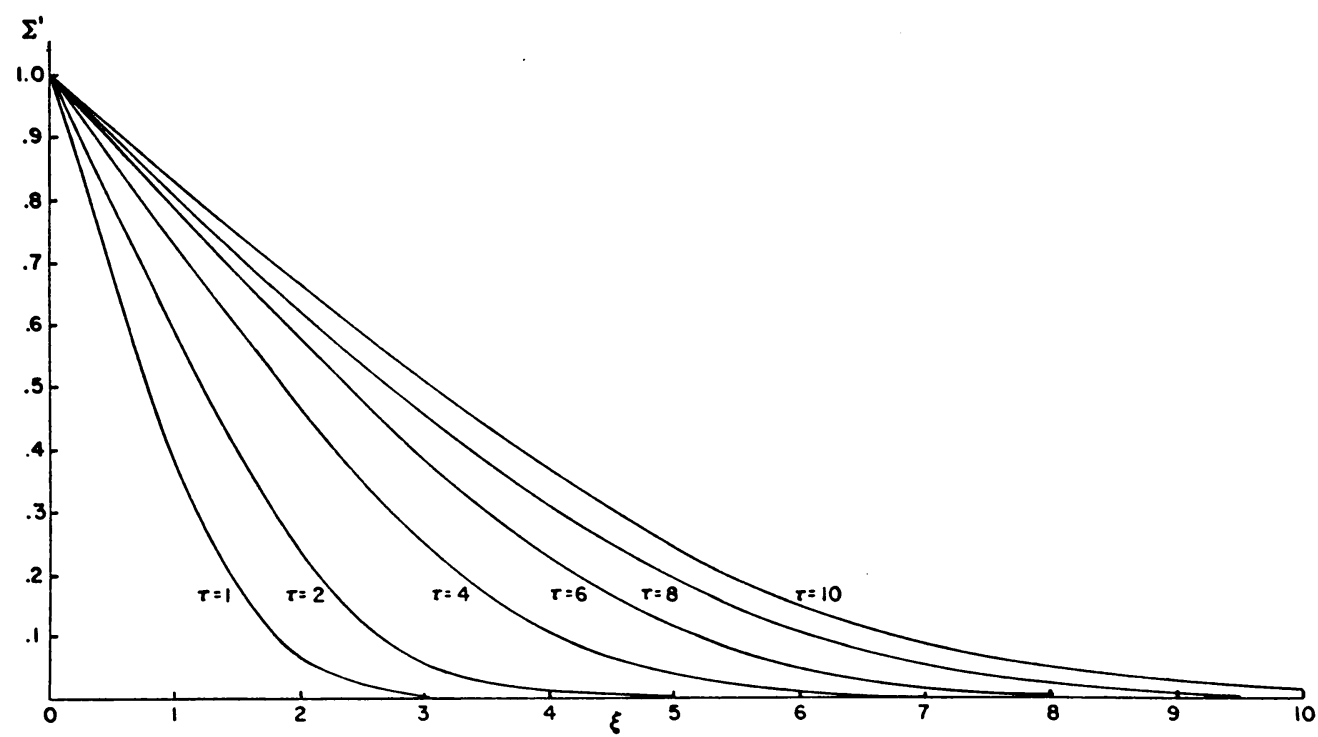

Fig. 6(b). Three parameter (1 elastic, 2 viscous). 
to decrease as $\tau$ increases and at each position in the rod the stress ultimately vanishes.

In Fig. 6(b) the stress distribution in the rod at various times, arising from an impulsively applied and subsequently maintained constant stress at the end of the rod, is illustrated. At each position in the rod $\Sigma^{\prime}$ ultimately approaches the value unity.

3. The three-parameter model with one viscous and two elastic elements. We now consider the remaining two true three-parameter models which are shown in Figs. 7(a) and $7(\mathrm{~b})$, these having two springs and one dashpot.

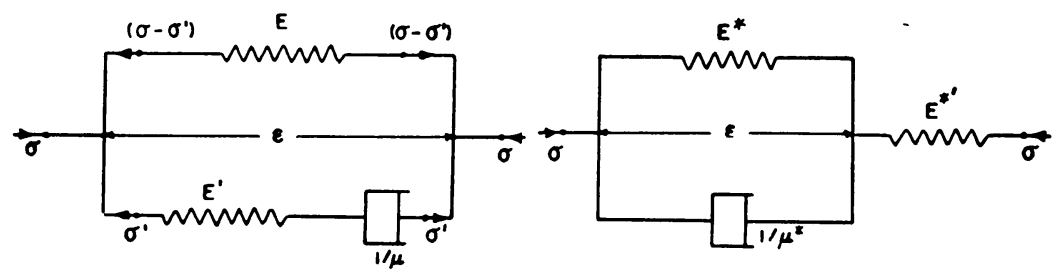

Fig. 7(a).

FIG. 7(b).

For the model of Fig. 7(a),

Hence, eliminating $\sigma^{\prime}$,

$$
\begin{aligned}
\epsilon_{t} & =\frac{\sigma_{t}^{\prime}}{E^{\prime}}+\mu \sigma^{\prime}, \\
\epsilon & =\frac{1}{E}\left(\sigma-\sigma^{\prime}\right) .
\end{aligned}
$$

In a similar manner we obtain the stress strain relation for the model of Fig. 7(b),

$$
E^{*} \epsilon+\frac{1}{\mu^{*}} \epsilon_{t}=\sigma\left(1+\frac{E^{*}}{E^{*^{\prime}}}\right)+\frac{\sigma_{t}}{E^{*^{\prime} \mu^{*}}} \cdot
$$

If we let $\mathrm{E}^{*} / E=E^{* \prime} / E^{\prime}=\left(\mu / \mu^{*}\right)^{1 / 2}=1+\left(E / E^{\prime}\right)$, then Eqs. (17) and (18) become identical so that we confine our attention to the model of Fig. 7(a). If we set $\sigma=\sigma_{0} H(t)$ in Eq. (17) and integrate we find that

$$
\epsilon=\frac{\sigma_{0}}{E}\left\{1-\frac{E^{\prime}}{\left(E+E^{\prime}\right)} \exp \left[-\frac{E E^{\prime} \mu t}{\left(E+E^{\prime}\right)}\right]\right\} H(t),
$$

so that the creep test with unloading is give by Eq. (2) with

$$
\varphi(\tau)=\left\{1-\frac{1}{(1+k)} \exp \left[\frac{-\tau}{(1+k)}\right]\right\},
$$

where $k=E / E^{\prime}$. This is depicted graphically in Fig. 8 for the particular case $k=1$.

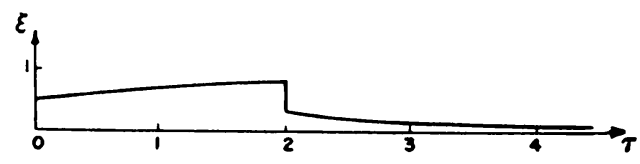

FIG. 8. 
For this model there is an instantaneous elastic strain of magnitude $\sigma_{0} /\left(E+E^{\prime}\right)=\sigma_{0} / E^{* \prime}$ when the stress is applied and the strain instantaneously falls by this same amount when the applied stress is removed, and asymptotically tends to zero. (This behavior is perhaps more apparent from Fig. 7(b), in which there is a spring of elastic constant $E^{* \prime}$ in series with a retarded elastic element.)

Eliminating $\sigma$ and $\epsilon$ from Eqs. (4), (5) and (17),

$$
\frac{\rho u_{t \ell t}}{E^{\prime}}+\rho \mu u_{t \imath}=u_{x x t}\left(1+\frac{E}{E^{\prime}}\right)+E_{\mu} u_{x x},
$$

and it may be verified that $\sigma$ and $v$ satisfy the same equation. The stress distributions $\boldsymbol{\Sigma}(\xi, \tau)$ and $\Sigma^{\prime}(\xi, \tau)$ for the problems previously considered for the other materials are determined for this material in Appendix C, Eqs. (C.20) and (C.19), respectively. From the prescribed condition on the end of the rod $\Sigma^{\prime}(0, \tau)=1$ and a simpler expression for $\Sigma(0, \tau)$ is given in Eq. (C.25), so that these provide a check on the accuracy of the computation of $\Sigma^{\prime}(\xi, \tau)$ and $\Sigma(\xi, \tau)$ from Eqs. (C.19) and (C.20).

$\Sigma$ and $\Sigma^{\prime}$ are plotted in Figs. $9(\mathrm{a})$ and $9(\mathrm{~b})$, respectively, against $\xi$ for different values

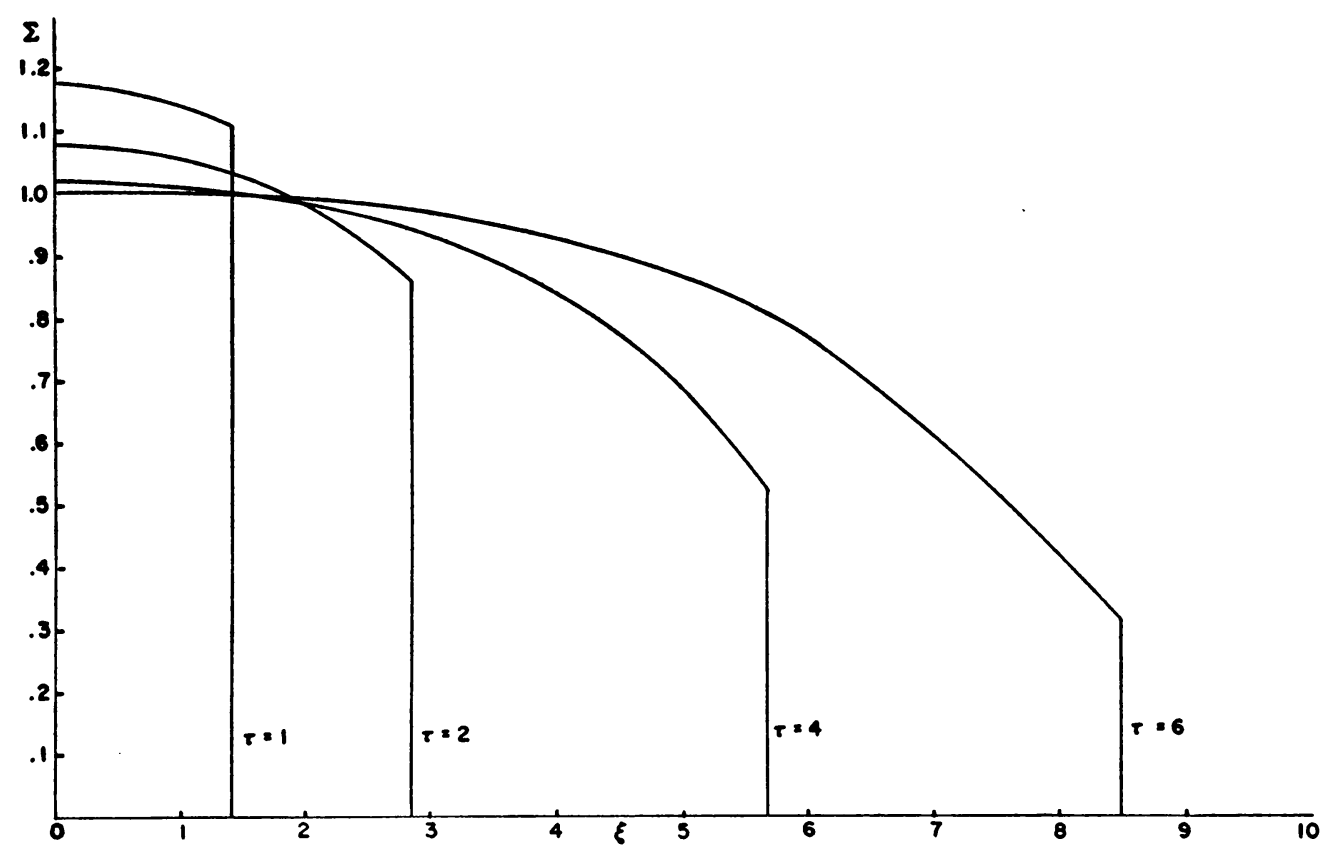

Fig. 9(a). Three parameter (2 elastic, 1 viscous).

of $\tau$ in the particular case $k=1$, so that the two elastic constants in the model are equal. Thus in Fig. 9(a) the stress distribution in the rod at various times, arising from an impulsively applied and subsequently maintained constant velocity at the end of the rod, is illustrated. A wave of stress discontinuity is propagated along the rod with constant velocity $(2 E / \rho)^{1 / 2}$ (or in the general case $\left.[(1+k) E / k \rho]^{1 / 2}=\left[\left(E+E^{\prime}\right) / \rho\right]^{1 / 2}\right)$, relative to the unstrained rod. Hence at time $\tau$ the portion of the rod for which $\xi>(2)^{1 / 2} \tau$ (or $\xi>(k+1)^{1 / 2} \tau / k^{1 / 2}$, in the general case) is unstressed. The magnitude of the stress discontinuity at $\xi=(2)^{1 / 2} \tau$, (or $\left.\xi=(k+1)^{1 / 2} \tau / k^{1 / 2}\right)$, is $(2)^{1 / 2} \exp (-\tau / 4)$, (or [ $(k+$ 
1) $\left./ k]^{1 / 2} \exp [-\tau / 2 k(k+1)]\right)$, so that it decreases exponentially as the wavefront advances along the rod. In the case considered, the stress at the end of the rod jumps to the value $(2)^{1 / 2}$ immediately after impact and then monotonically decreases, ultimately approaching the value unity. The stress at each position in the rod also ultimately approaches the value unity.

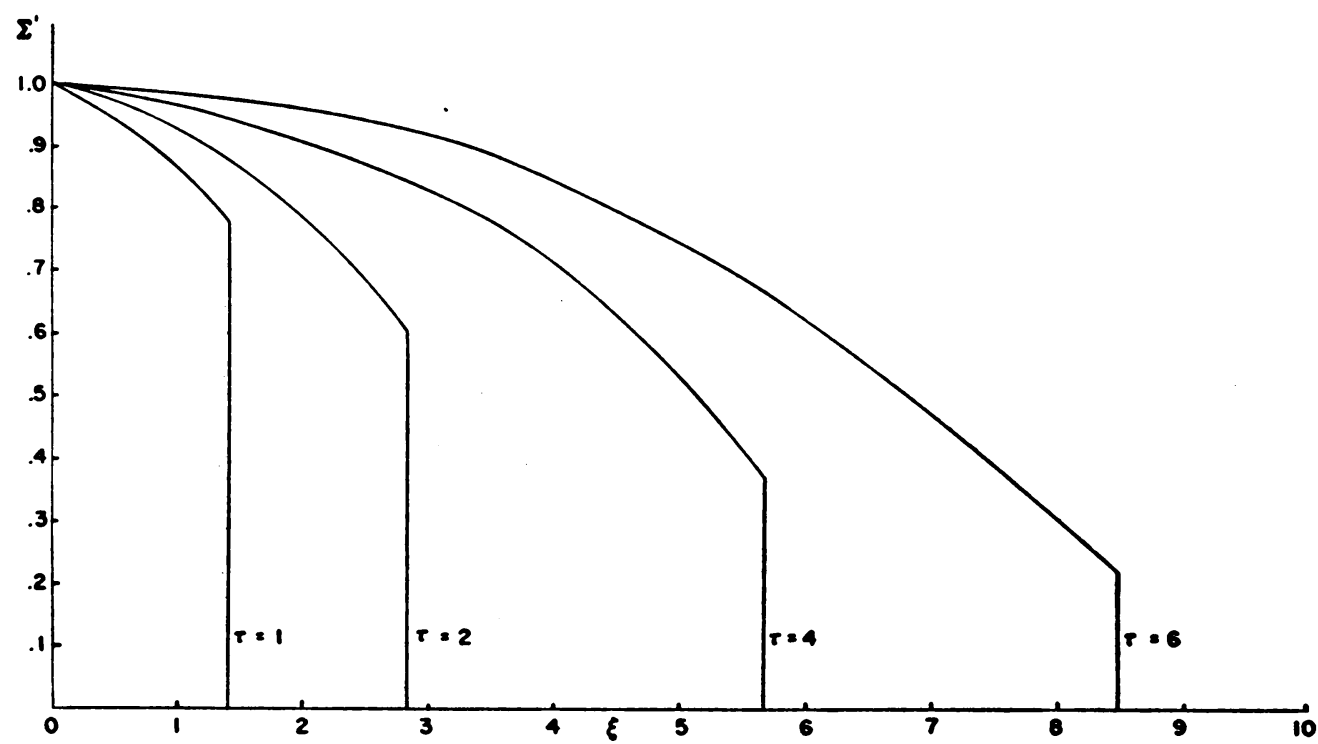

FIG. 9(b). Three parameter (2 elastic, 1 viscous).

In Fig. 9(b) the stress distribution in the rod at various times, due to an impulsively applied and subsequently maintained constant stress at the end of the rod, is illustrated. Here again a wave of stress discontinuity is propagated along the rod, the constant velocity of propagation being the same as before. In this case the magnitude of the stress discontinuity at $\xi=(2)^{1 / 2} \tau$, (or at $\xi=(k+1)^{1 / 2} \tau / k^{1 / 2}$ in the general case), is $\exp (-\tau / 4)$, (or $\exp [-\tau / 2 k(k+1]$ ), so that again it decreases exponentially as the wavefront advances along the rod. The stress at each position in the rod ultimately approaches the value unity.

4. Concluding remarks. We have been concerned with the stress distributions in rods, of Voigt material and of visco-elastic materials with three-parameter models, impulsively subjected to constant applied velocity or constant applied stress at the end of the rod. We have seen that there are two basic types of true three-parameter models, namely those with one spring and two dashpots and those with one dashpot and two springs. In a subsequent paper it is intended to compare the stress distributions $\Sigma(\xi, \tau)$ and $\Sigma^{\prime}(\xi, \tau)$ for seven different visco-elastic materials; elastic, viscous, Maxwell, Voigt, the two basic three-parameter models considered here and also the four-parameter model considered by Glauz and Lee ${ }^{2}$. With this in mind we chose the model of Fig. 4(a), in preference to that of Fig. 4(b), since the ultimate behavior may then be compared with that of a dashpot with viscous constant $\mu$. Similarly we chose the model of Fig. 7 (a) since its ultimate behavior may be compared with that of the Voigt model considered in Sec. 1. 
Acknowledgement. The author wishes to express his gratitude to Professor E. H. Lee for his advice and encouragement during the preparation of this work, and also to various members of the computing staff at Brown University for carrying out the numerical integrations.

Appendix A. From Eq. (7) it is found that $V(\xi, \tau)$ satisfies the equation

$$
V_{\tau \tau}=V_{\xi \xi}+V_{\xi \xi \tau} \text {. }
$$

Also, from Eq. (4),

$$
V_{\tau}=-\Sigma_{\xi} .
$$

In addition there are the boundary conditions,

Applying the Laplace transform,

$$
\begin{gathered}
V(\infty, \tau)=0=\Sigma(\infty, \tau), \\
V(0, \tau)=1 .
\end{gathered}
$$

$$
L\{f(\xi, \tau)\}=\int_{0}^{\infty} e^{-a \tau} f(\xi, \tau) d \tau,
$$

to Eqs. (A.1-A.3) and remembering that the rod is initially unstressed, unstrained and at rest, we obtain

$$
\begin{gathered}
q^{2} L\{V\}=(1+q)[L\{V\}]_{\xi \xi}=(1+q) L\left\{V_{\xi \xi}\right\} \\
q L\{V\}=-[L\{\Sigma\}]_{\xi}=-L\left\{\Sigma_{\xi}\right\}
\end{gathered}
$$

and

$$
\begin{gathered}
L\{V(\infty, \tau)\}=0=L\{\Sigma(\infty, \tau)\}, \\
L\{V(0, \tau)\}=\frac{1}{q} .
\end{gathered}
$$

Solving the ordinary differential Equation (A.5) for $L\{V(\xi, \tau)\}$ and applying the boundary conditions (A.7), we obtain

$$
L\{V(\xi, \tau)\}=\frac{1}{q} \exp \left[\frac{-\xi q}{(1+q)^{1 / 2}}\right] .
$$

From Eq. (A.6) and the boundary condition in (A.7),

$$
L\{\Sigma(\xi, \tau)\}=\frac{(1+q)^{1 / 2}}{q} \exp \left[\frac{-\xi q}{(1+q)^{1 / 2}}\right] .
$$

In carrying out the inversions of $L\{V\}$ and $L\{\Sigma\}$ we make use of the following general result for Laplace transforms which is valid under certain conditions. ${ }^{5}$

If

$$
F(q)=L\{f(\tau)\} \quad \text { and } \quad \frac{1}{g(q)} \exp [-\eta h(q)]=L\{\varphi(\tau, \eta)\}
$$

'H. S. Carslaw and J. C. Jaeger, Operational methods in applied mathematics, Oxford University Press, London, 1947, p. 259. 
then

$$
\frac{1}{g(q)} F[h(q)]=L\left\{\int_{0}^{\infty} \varphi(\tau, \eta) f(\eta) d \eta\right\}
$$

Now ${ }^{\circ}$,

$$
\frac{1}{(q)^{1 / 2}} \exp \left[-\xi(q)^{1 / 2}\right]=L\left\{\frac{\exp \left(-\xi^{2} / 4 \tau\right)}{(\pi \tau)^{1 / 2}}\right\}
$$

and

$$
\frac{1}{(q)^{1 / 2}} \exp \left(-\frac{\eta}{q}\right)=L\left\{\frac{\cos \left[2(\eta \tau)^{1 / 2}\right]}{(\pi \tau)^{1 / 2}}\right\}
$$

so that

$$
\frac{1}{(q)^{1 / 2}} \exp \left[-\eta\left(q+\frac{1}{q}-2\right)\right]=L\left\{\frac{\cos \left[2(\eta)^{1 / 2}(\tau-\eta)^{1 / 2}\right]}{(\pi)^{1 / 2}(\tau-\eta)^{1 / 2}} e^{2 \eta} H(\tau-\eta)\right\} .
$$

Hence, from the result of (A.10), for $q>1$,

$\frac{1}{(q-1)} \exp \left[\frac{-\xi(q-1)}{(q)^{1 / 2}}\right]=L\left\{\frac{1}{\pi} \int_{0}^{\tau} \frac{\cos \left[2(\eta)^{1 / 2}(\tau-\eta)^{1 / 2}\right]}{(\eta)^{1 / 2}(\tau-\eta)^{1 / 2}} \exp \left(2 \eta-\frac{\xi^{2}}{4 \eta}\right) d \eta\right\}$.

Applying the shift theorem we obtain from Eq. (A.8),

$$
\Sigma^{\prime}(\xi, \tau)=V(\xi, \tau)=\frac{e^{-\tau}}{\pi} \int_{0}^{\tau} \frac{\cos \left[2(\eta)^{1 / 2}(\tau-\eta)^{1 / 2}\right]}{(\eta)^{1 / 2}(\tau-\eta)^{1 / 2}} \exp \left(2 \eta-\frac{\xi^{2}}{4 \eta}\right) d \eta .
$$

Further,

$$
\exp \left(-\frac{\eta}{q}\right)=L\left\{\delta(\tau)-\left(\frac{\eta}{\tau}\right)^{1 / 2} J_{1}\left[2(\eta \tau)^{1 / 2}\right]\right\}
$$

where $\delta(\tau)$ is the delta function. Thus,

$$
\begin{array}{r}
\exp \left[-\eta\left(q+\frac{1}{q}-2\right)\right]=L\left\{e ^ { 2 \eta } \left[\delta(\tau-\eta)-\frac{(\eta)^{1 / 2}}{(\tau-\eta)^{1 / 2}} J_{1}\left\{2(\eta)^{1 / 2}(\tau-\eta)^{1 / 2}\right\}\right.\right. \\
\cdot \\
\cdot H(\tau-\eta)]\} .
\end{array}
$$

From Eqs. (A.10) and (A.11) it then follows that, for $q>1$,

$$
\begin{aligned}
\frac{(q)^{1 / 2}}{(q-1)} \exp \left[\frac{-\xi(q-1)}{(q)^{1 / 2}}\right]= & L\left\{\frac{\exp \left(2 \tau-\xi^{2} / 4 \tau\right)}{(\pi \tau)^{1 / 2}}\right. \\
& \left.-\int_{0}^{\tau} \frac{J_{1}\left[2(\eta)^{1 / 2}(\tau-\eta)^{1 / 2}\right]}{(\pi)^{1 / 2}(\tau-\eta)^{1 / 2}} \exp \left(2 \eta-\frac{\xi^{2}}{4 \eta}\right) d \eta\right\} .
\end{aligned}
$$

${ }^{6} \mathrm{R}$. V. Churchill, Modern operational mathematics in engineering, McGraw-Hill Book Co., Inc., New York, 1944, p. 299. 
Applying the shift theorem we obtain from Eq. (A.9),

$$
\begin{aligned}
\Sigma(\xi, \tau)=\frac{e^{\tau}}{(\pi)^{1 / 2}}\left\{\frac{\exp \left(-\xi^{2} / 4 \tau\right)}{(\tau)^{1 / 2}}\right. & \\
& \left.\quad-\int_{0}^{\tau} \frac{J_{1}\left[2(\eta)^{1 / 2}(\tau-\eta)^{1 / 2}\right]}{(\tau-\eta)^{1 / 2}} \exp \left[-2(\tau-\eta)-\frac{\xi^{2}}{4 \eta}\right] d \eta\right\} .
\end{aligned}
$$

Also, since

$$
L\{\Sigma(0, \tau)\}=\frac{(1+q)^{1 / 2}}{q}=\frac{(q+1)^{1 / 2}}{[(q+1)-1]}
$$

we find that

$$
\Sigma(0, \tau)=\left\{\frac{e^{-\tau}}{(\pi \tau)^{1 / 2}}+\operatorname{erf}\left[(\tau)^{1 / 2}\right]\right\}
$$

and

$$
\frac{d}{d \tau}\{\Sigma(0, \tau)\}=\frac{-e^{-\tau}}{2(\pi)^{1 / 2}(\tau)^{3 / 2}} .
$$

Appendix B. Equation (15) in dimensionless form becomes

$$
V_{\tau}+(1+\lambda) V_{r \tau}=V_{\xi \xi}+\lambda V_{\xi \xi \tau} .
$$

Equations (A.2) and (A.3) still hold and applying the Laplace transform we obtain

$$
q[1+(1+\lambda) q] L\{V\}=(1+\lambda q) L\left\{V_{\xi \xi}\right\},
$$

together with Eqs. (A.6) and (A.7). Solving Eq. (B.2) for $L\{V(\xi, \tau)\}$ and applying the boundary conditions of (A.7),

$$
L\{V(\xi, \tau)\}=\frac{1}{q} \exp \left\{-\frac{\xi q[1+(1+\lambda) q]^{1 / 2}}{(1+\lambda q)^{1 / 2}}\right\} .
$$

From Eq. (A.6) and the boundary condition in (A.7),

$$
L\{\Sigma(\xi, \tau)\}=\frac{(1+\lambda q)^{1 / 2}}{(q)^{1 / 2}[1+(1+\lambda) q]^{1 / 2}} \exp \left\{-\frac{\xi q[1+(1+\lambda) q]^{1 / 2}}{(1+\lambda q)^{1 / 2}}\right\} .
$$

The inversion of $L\{\Sigma(\xi, \tau)\}$ will be carried out in a manner similar to that of Appen$\operatorname{dix}$ A.

$$
\exp \left[-\eta\left\{q+\frac{1}{\lambda^{2}(1+\lambda) q}-\frac{(2+\lambda)}{\lambda(1+\lambda)}\right\}\right]=L\{\psi(\tau, \eta)\}
$$

where

$$
\begin{aligned}
& \psi(\tau, \eta)=\left\{\exp \left[\frac{(2+\lambda) \eta}{\lambda(1+\lambda)}\right][\delta(\tau-\eta)\right. \\
&\left.\left.\quad-\frac{(\eta)^{1 / 2}}{\lambda(1+\lambda)^{1 / 2}(\tau-\eta)^{1 / 2}} J_{1}\left\{\frac{2(\eta)^{1 / 2}(\tau-\eta)^{1 / 2}}{\lambda(1+\lambda)^{1 / 2}}\right\} H(\tau-\eta)\right]\right\} .
\end{aligned}
$$


Hence, using the shift theorem,

$$
\exp \left\{-\frac{\eta \lambda q[1+(1+\lambda) q]}{(1+\lambda)(1+\lambda q)}\right\}=L\left\{e^{-r / \lambda} \psi(\tau, \eta)\right\}
$$

But,

$$
\frac{1}{(q)^{1 / 2}} \exp \left[-\frac{\xi(1+\lambda)^{1 / 2}(q)^{1 / 2}}{(\lambda)^{1 / 2}}\right]=L\left\{\frac{1}{(\pi \tau)^{1 / 2}} \exp \left[-\frac{(1+\lambda) \xi^{2}}{4 \lambda \tau}\right]\right\} .
$$

Hence, from the result of (A.10), we obtain

$$
\begin{aligned}
& \Sigma(\xi, \tau)=\frac{1}{(\lambda)^{1 / 2}(1+\lambda)(\pi)^{1 / 2}} \exp \left[\frac{\tau}{\lambda(1+\lambda)}\right]\left\{\frac{\lambda(1+\lambda)^{1 / 2}}{(\tau)^{1 / 2}} \exp \left[-\frac{(1+\lambda) \xi^{2}}{4 \lambda \tau}\right]\right. \\
& \left.-\int_{0}^{\tau} J_{1}\left[\frac{2(\eta)^{1 / 2}(\tau-\eta)^{1 / 2}}{\lambda(1+\lambda)^{1 / 2}}\right] \exp \left[-\frac{(2+\lambda)(\tau-\eta)}{\lambda(1+\lambda)}-\frac{(1+\lambda) \xi^{2}}{4 \lambda \eta}\right] \frac{d \eta}{(\tau-\eta)^{1 / 2}}\right\} .
\end{aligned}
$$

We now carry out the inversion of $L\{V(\xi, \tau)\}$.

$$
\frac{1}{q} \exp \left[-\eta\left\{q+\frac{1}{\lambda^{2}(1+\lambda) q}-\frac{(2+\lambda)}{\lambda(1+\lambda)}\right\}\right]=L\{\chi(\tau, \eta)\},
$$

where

$$
\chi(\tau, \eta)=\exp \left[\frac{\eta(2+\lambda)}{\lambda(1+\lambda)}\right] J_{0}\left[\frac{2(\eta)^{1 / 2}(\tau-\eta)^{1 / 2}}{\lambda(1+\lambda)^{1 / 2}}\right] H(\tau-\eta) .
$$

Hence, using the shift theorem,

$$
\frac{\lambda}{(1+\lambda q)} \exp \left\{\frac{-\eta \lambda q[1+(1+\lambda) q]}{(1+\lambda)(1+\lambda q)}\right\}=L\left\{e^{-\tau / \lambda} \chi(\tau, \eta)\right\}
$$

From (B.6) and (B.10) we find that

$$
\begin{array}{r}
\frac{\lambda^{2}[1+(1+\lambda) q]}{(1+\lambda q)} \exp \left\{\frac{-\eta \lambda q[1+(1+\lambda) q]}{(1+\lambda)(1+\lambda q)}\right\}=L\left\{e^{-\tau / \lambda}[\lambda(1+\lambda) \psi(\tau, \eta)\right. \\
-\chi(\tau, \eta)]\} .
\end{array}
$$

But,

$$
\frac{1}{q} \exp \left[\frac{-\xi(1+\lambda)^{1 / 2}(q)^{1 / 2}}{(\lambda)^{1 / 2}}\right]=L\left\{\operatorname{erfc}\left[\frac{\xi(1+\lambda)^{1 / 2}}{2(\lambda \tau)^{1 / 2}}\right]\right\} .
$$

Hence, from (B.11) and (B.12), using the result of (A.10),

$$
\Sigma^{\prime}(\xi, \tau)=V(\xi, \tau)=e^{-\tau / \mathrm{\lambda}} \int_{0}^{\infty}\left[\psi(\tau, \eta)-\frac{\chi(\tau, \eta)}{\lambda(1+\lambda)}\right] \operatorname{erfc}\left[\frac{\xi(1+\lambda)^{1 / 2}}{2(\lambda \eta)^{1 / 2}}\right] d \eta
$$


Thus, finally,

$$
\begin{gathered}
\Sigma^{\prime}(\xi, \tau)=\exp \left[\frac{\tau}{\lambda(1+\lambda)}\right]\left(\operatorname{erfc}\left[\frac{\xi(1+\lambda)^{1 / 2}}{2(\lambda \tau)^{1 / 2}}\right]\right. \\
-\frac{1}{\lambda(1+\lambda)} \int_{0}^{\tau}\left\{J_{0}\left[\frac{2(\eta)^{1 / 2}(\tau-\eta)^{1 / 2}}{\lambda(1+\lambda)^{1 / 2}}\right]+\frac{(1+\lambda)^{1 / 2}(\eta)^{1 / 2}}{(\tau-\eta)^{1 / 2}} J_{1}\left[\frac{2(\eta)^{1 / 2}(\tau-\eta)^{1 / 2}}{\lambda(1+\lambda)^{1 / 2}}\right]\right\} \\
\left.\cdot \exp \left[\frac{-(2+\lambda)(\tau-\eta)}{\lambda(1+\lambda)}\right] \operatorname{erfc}\left[\frac{\xi(1+\lambda)^{1 / 2}}{2(\lambda \eta)^{1 / 2}}\right] d \eta\right) .
\end{gathered}
$$

Appendix C. Equation (21) for the velocity becomes, in dimensionless form,

$$
V_{\tau \tau}+k V_{\tau \tau \tau}=V_{\xi \xi}+(1+k) V_{\xi \xi \tau} \text {. }
$$

Equations (A.2) and (A.3) again hold and applying the Laplace transform we obtain

$$
q^{2}(1+k q) L\{V\}=[1+(1+k) q] L\left\{V_{\xi \xi}\right\},
$$

together with Eqs. (A.6) and (A.7). Solving Eq. (C.2) for $L\{V(\xi, \tau)\}$ and applying the boundary conditions of (A.7),

$$
L\{V(\xi, \tau)\}=\frac{1}{q} \exp \left\{\frac{-\xi q(1+k q)^{1 / 2}}{[1+(1+k) q]^{1 / 2}}\right\} .
$$

From Eq. (A.6) and the boundary condition in (A.7),

$$
L\{\Sigma(\xi, \tau)\}=\frac{[1+(1+k) q]^{1 / 2}}{q(1+k q)^{1 / 2}} \exp \left\{\frac{-\xi q(1+k q)^{1 / 2}}{[1+(1+k) q]^{1 / 2}}\right\}
$$

Let

$$
\exp \left\{\frac{-\xi q(1+k q)^{1 / 2}}{[1+(1+k) q]^{1 / 2}}\right\}=L\{A(\xi, \tau)\}
$$

so that $A(\xi, \tau)$ is the dimensionless acceleration. Then,

$$
V(\xi, \tau)=\int_{0}^{\tau} A(\xi, \zeta) d \zeta
$$

and

$$
\Sigma(\xi, \tau)=\int_{\xi}^{\infty} A(\zeta, \tau) d \zeta
$$

Let

$$
\alpha_{-}=\frac{(2 k+1)}{2 k(k+1)}, \quad \beta=\frac{1}{2 k(k+1)}, \quad \omega=\frac{\xi(k)^{1 / 2}}{(1+k)^{1 / 2}}
$$

Then,

$$
\exp \left[-\omega q \frac{(q+\alpha+\beta)^{1 / 2}}{(q+\alpha-\beta)^{1 / 2}}\right]=\exp \left\{-\frac{\xi q(1+k q)^{1 / 2}}{[1+(1+k) q]^{1 / 2}}\right\}
$$


Now,

$$
\begin{gathered}
\exp \left[-\omega(q-\alpha) \frac{(q+\beta)^{1 / 2}}{(q-\beta)^{1 / 2}}\right]=\exp (-\omega q) \exp [-\omega(\alpha-2 \beta)] \exp \left[-\omega\left\{\left[\left(q^{2}-\beta^{2}\right)^{1 / 2}\right.\right.\right. \\
\left.\left.-q+\beta]-\frac{2 \beta(\alpha-\beta)}{\left[\left(q^{2}-\beta^{2}\right)^{1 / 2}-q+\beta\right]}\right\}\right] .
\end{gathered}
$$

Also,

$$
\begin{aligned}
\left\{\exp \left[-\eta\left(q^{2}-\beta^{2}\right)^{1 / 2}\right]-\exp (-\eta q)\right\} & \\
\quad & L\left\{\frac{\beta \eta}{\left(\tau^{2}-\eta^{2}\right)^{1 / 2}} I_{1}\left[\beta\left(\tau^{2}-\eta^{2}\right)^{1 / 2}\right] H(\tau-\eta)\right\},
\end{aligned}
$$

so that

$$
\begin{aligned}
\exp \left[-\eta\left\{\left(q^{2}-\beta^{2}\right)^{1 / 2}-q\right.\right. & +\beta\}]=L\left\{e^{-\beta \eta}[\delta(\tau)\right. \\
& \left.\left.+\frac{\beta \eta}{(\tau)^{1 / 2}(\tau+2 \eta)^{1 / 2}} I_{1}\left\{\beta(\tau)^{1 / 2}(\tau+2 \eta)^{1 / 2}\right\} H(\tau)\right]\right\} .
\end{aligned}
$$

From (C.12) and (A.10) it follows that, if $F(q)=L\{f(\tau)\}$,

$$
\begin{aligned}
& F\left[\left(q^{2}-\beta^{2}\right)^{1 / 2}-q+\beta\right]=L\{F(\beta) \delta(\tau) \\
& \left.\quad+\beta \int_{0}^{\infty} e^{-\beta \eta} \eta \frac{I_{1}\left[\beta(\tau)^{1 / 2}(\tau+2 \eta)^{1 / 2}\right]}{(\tau)^{1 / 2}(\tau+2 \eta)^{1 / 2}} f(\eta) d \eta\right\} .
\end{aligned}
$$

But,

$$
\begin{aligned}
\exp \left[-\omega\left\{q-\frac{1}{k(k+1)^{2} q}\right\}\right]=L\{\delta(\tau-\omega) & \\
& \left.\quad+\frac{(\omega)^{1 / 2}}{(k)^{1 / 2}(k+1)(\tau-\omega)^{1 / 2}} I_{1}\left[\frac{2(\omega)^{1 / 2}(\tau-\omega)^{1 / 2}}{(k)^{1 / 2}(k+1)}\right] H(\tau-\omega)\right\} .
\end{aligned}
$$

Thus, since

$$
\begin{gathered}
2 \beta(\alpha-\beta)=\frac{1}{k(k+1)^{2}}, \\
\exp \left[-\omega\left\{\left[\left(q^{2}-\beta^{2}\right)^{1 / 2}-q+\beta\right]-\frac{2 \beta(\alpha-\beta)}{\left[\left(q^{2}-\beta^{2}\right)^{1 / 2}-q+\beta\right]}\right\}\right] \\
=L\left\{\exp [\omega(2 \alpha-3 \beta)] \delta(\tau)+\beta \omega e^{-\beta \omega} \frac{I_{1}\left[\beta(\tau)^{1 / 2}(\tau+2 \omega)^{1 / 2}\right]}{(\tau)^{1 / 2}(\tau+2 \omega)^{1 / 2}}\right. \\
+\beta \int_{\omega}^{\infty} e^{-\beta \eta} \eta \frac{I_{1}\left[\beta(\tau)^{1 / 2}(\tau+2 \eta)^{1 / 2}\right]}{(\tau)^{1 / 2}(\tau+2 \eta)^{1 / 2}} \\
\left.\cdot \frac{(\omega)^{1 / 2}}{(k)^{1 / 2}(1+k)} I_{1}\left[\frac{2(\omega)^{1 / 2}(\eta-\omega)^{1 / 2}}{(k)^{1 / 2}(1+k)}\right] \frac{d \eta}{(\eta-\omega)^{1 / 2}}\right\} .
\end{gathered}
$$


Hence, from (C.10) and (C.15),

$$
\begin{gathered}
\exp \left[-\omega(q-\alpha) \frac{(q+\beta)^{1 / 2}}{(q-\beta)^{1 / 2}}\right]=L\{\exp [\omega(\alpha-\beta)] \delta(\tau-\omega) \\
+\beta \omega \exp [-\omega(\alpha-\beta)] \frac{I_{1}\left[\beta\left(\tau^{2}-\omega^{2}\right)^{1 / 2}\right]}{\left(\tau^{2}-\omega^{2}\right)^{1 / 2}} H(\tau-\omega) \\
+\beta \exp [-\omega(\alpha-\beta)] H(\tau-\omega) \int_{0}^{\infty} e^{-\beta \eta}(\eta+\omega) \frac{I_{1}\left[\beta\left\{(\tau+\eta)^{2}-(\eta+\omega)^{2}\right\}^{1 / 2}\right]}{\left[(\tau-\eta)^{2}-(\eta+\omega)^{2}\right]^{1 / 2}} \\
\left.\quad \frac{(\omega)^{1 / 2}}{(k)^{1 / 2}(1+k)} I_{1}\left[\frac{2(\omega \eta)^{1 / 2}}{(k)^{1 / 2}(1+k)}\right] \frac{d \eta}{(\eta)^{1 / 2}}\right\} .
\end{gathered}
$$

Thus finally, using the shift theorem, from (C.5), (C.8) and (C.9)

$$
\begin{aligned}
& A(\xi, \tau)=\exp \left[\frac{-(2 k+1) \tau}{2 k(k+1)}\right]\left\{\exp \left[\frac{(k)^{1 / 2} \xi}{(k+1)^{3 / 2}}\right] \delta\left[\tau-\frac{(k)^{1 / 2} \xi}{(k+1)^{1 / 2}}\right]\right. \\
& +\frac{1}{2(k)^{1 / 2}(k+1)^{3 / 2}} \exp \left[-\frac{(k)^{1 / 2} \xi}{(k+1)^{3 / 2}}\right] H\left[\tau-\frac{(k)^{1 / 2} \xi}{(k+1)^{1 / 2}}\right] \\
& \cdot\left[\frac{\xi}{\left\{\tau^{2}-\frac{k \xi^{2}}{(k+1)}\right\}^{1 / 2}} I_{1}\left[\frac{\left\{\tau^{2}-\frac{k \xi^{2}}{(k+1)}\right\}^{1 / 2}}{2 k(k+1)}\right]\right. \\
& +\int_{0}^{\infty} \exp \left[\frac{-\eta}{2(k)^{1 / 2} \cdot(k+1)^{3 / 2}}\right] \frac{(\eta+\xi)(\xi)^{1 / 2}}{(k+1)^{3 / 2}(\eta)^{1 / 2}} I_{1}\left[\frac{2(\xi \eta)^{1 / 2}}{(k+1)^{3 / 2}}\right] \\
& \cdot I_{1}\left[\frac{\left.\left\{\tau+\frac{(k)^{1 / 2} \eta}{(k+1)^{1 / 2}}\right]^{2}-\frac{k(\xi+\eta)^{2}}{(k+1)}\right\}^{1 / 2}}{2 k(k+1)}\right] \\
& \left.\cdot \frac{d \eta}{\left\{\left[\tau+\frac{(k)^{1 / 2} \eta}{(k+1)^{1 / 2}}\right]^{2}-\frac{k(\xi+\eta)^{2}}{(k+1)}\right\}^{1 / 2}}\right] \text {. }
\end{aligned}
$$

If we let

$$
B(\xi, \tau)=A(\xi, \tau)-\exp \left[\frac{-(2 k+1) \tau}{2 k(k+1)}+\frac{(k)^{1 / 2} \xi}{(k+1)^{3 / 2}}\right] \delta\left[\tau-\frac{(k)^{1 / 2} \xi}{(k+1)^{1 / 2}}\right],
$$

then from (C.6), (C.7) and (C.17),

$\Sigma^{\prime}(\xi, \tau)=V(\xi, \tau)=\left\{\exp \left[\frac{-\xi}{2(k)^{1 / 2}(k+1)^{3 / 2}}\right]\right.$

$$
\left.+\int_{\left[(k)^{2 / 2} \xi\right] /\left[(k+1)^{1 / 2]}\right.}^{\tau} B(\xi, \zeta) d \zeta\right\} H\left[\tau-\frac{(k)^{1 / 2} \xi}{(k+1)^{1 / 2}}\right],
$$

and

$$
\begin{aligned}
\Sigma(\xi, \tau)=\left\{\frac{(k+1)^{1 / 2}}{(k)^{1 / 2}} \exp \right. & {\left[\frac{-\tau}{2 k(k+1)}\right] } \\
& \left.+\int_{\xi}^{\left[(k+1)^{2 / 2}+1 /(k)^{1 / 3}\right.} B(\zeta, \tau) d \zeta\right\} H\left[\tau-\frac{(k)^{1 / 2} \xi}{(k+1)^{1 / 2}}\right] .
\end{aligned}
$$


From (C.17) and (C.18) we obtain

$$
\begin{aligned}
& \lim _{\left\{r \rightarrow\left\{(k)^{1 / 2} \xi 1 / /(k+1)^{1 / \imath}\right\}+1\right.}\{B(\xi, \tau)\}=\frac{(4 k+1) \xi}{8(k)^{3 / 2}(k+1)^{3 / 2}} \exp \left[\frac{-\xi}{2(k)^{1 / 2}(k+1)^{3 / 2}}\right] \\
& =\lim _{\left\{\tau \rightarrow \left[(k)^{2 / \rho} \mid /\left[(k+1)^{1 / 2}\right]+1\right.\right.}\left\{\frac{\partial \Sigma^{\prime}}{\partial \tau}(\xi, \tau)\right\},
\end{aligned}
$$

and

$$
\begin{aligned}
& \lim _{\left\{\xi \rightarrow \left((k+1)^{2 / \imath \tau 1 /(k) 1 / \ell-1}\right.\right.}\{B(\xi, \tau)\}=\frac{(4 k+1) \tau}{8(k)^{2}(k+1)^{2}} \exp \left[\frac{-\tau}{2 k(k+1)}\right] \\
& =\lim _{\left[\xi \rightarrow \left[(k+1)^{2 / \imath+\tau] /(k)^{2 /:-1}}\right.\right.}\left\{-\frac{\partial \Sigma}{\partial \xi}(\xi, \tau)\right\} \text {. }
\end{aligned}
$$

Also, from (C.19) and (C.20),

$$
\begin{aligned}
\lim _{\left\{r \rightarrow \mid(k)^{2 / 1} \xi \xi /(k+1)^{2 / 1}\right]_{+1}} & \left\{\frac{\partial \Sigma}{\partial \tau}(\xi, \tau)\right\} \\
= & \frac{\left[(4 k+1) \xi-4(k)^{1 / 2}(k+1)^{3 / 2}\right]}{8(k)^{2}(k+1)^{2}} \exp \left[\frac{-\xi}{2(k)^{1 / 2}(k+1)^{3 / 2}}\right],
\end{aligned}
$$

and

$$
\lim _{\left\{\xi \rightarrow \left[(k+1)^{2 / s_{\tau} /(k)^{2 / 8-1}}\right.\right.}\left\{\frac{\partial \Sigma^{\prime}}{\partial \xi}(\xi, \tau)\right\}=\frac{[(4 k+1) \tau+4 k(k+1)]}{8(k)^{3 / 2}(k+1)^{5 / 2}} \exp \left[\frac{-\tau}{2 k(k+1)}\right] .
$$

Further,

$$
\begin{aligned}
& \lim _{\left\{\xi \rightarrow \left[(k+1)^{1 / 2+\tau] /(k)^{1 / s-1}}\right.\right.}\left\{\Sigma^{\prime}(\xi, \tau)\right\}=\exp \left[\frac{-\tau}{2 k(k+1)}\right], \\
& \lim _{\left\{\xi \rightarrow \left[(k+1)^{1 / 2 \tau] /(k)^{1 / 2-1}}\right.\right.}\{\Sigma(\xi, \tau)\}=\frac{(k+1)^{1 / 2}}{(k)^{1 / 2}} \exp \left[\frac{-\tau}{2 k(k+1)}\right] .
\end{aligned}
$$

From Eq. (C.4),

$$
\begin{aligned}
L\{\Sigma(0, \tau)\}= & \frac{[1+(1+k) q]^{1 / 2}}{q(1+k q)^{1 / 2}} \\
= & \frac{\left[(1+k)+\frac{1}{q}\right]}{(k)^{1 / 2}(1+k)^{1 / 2}} \frac{1}{\left\{\left[q+\frac{(2 k+1)}{2 k(k+1)}\right]^{2}-\frac{1}{4 k^{2}(k+1)^{2}}\right\}^{1 / 2}},
\end{aligned}
$$

so that

$$
\begin{aligned}
\Sigma(0, \tau)=\frac{(1+k)^{1 / 2}}{(k)^{1 / 2}}\{ & \exp \left[\frac{-(2 k+1) \tau}{2 k(k+1)}\right] I_{0}\left[\frac{\tau}{2 k(k+1)}\right] \\
& \left.+\frac{1}{(1+k)} \int_{0}^{\tau} \exp \left[\frac{-(2 k+1) \eta}{2 k(k+1)}\right] I_{0}\left[\frac{\eta}{2 k(k+1)}\right] d \eta\right\} .
\end{aligned}
$$

In particular, $\Sigma(0, \infty)=1$. 